\title{
Barrett's Esophagus Without Dysplasia: Wait or Ablate?
}

\author{
Stuart Jon Spechler
}

Published online: 21 April 2011

(C) Springer Science+Business Media, LLC (Outside the USA) 2011

In Barrett's esophagus, metaplastic columnar epithelium that is predisposed to malignancy replaces the stratified squamous epithelium that normally lines the distal esophagus [1]. Barrett's metaplasia develops as a result of chronic esophageal inflammation caused by the gastroesophageal reflux of noxious material like acid and bile. Endoscopic ablation of this metaplasia has been proposed as a way to prevent cancer. Recent attention has focused on radiofrequency ablation (RFA), which uses endoscopic guidance to position a balloon with a circumferential array of electrodes (the $\mathrm{HALO}^{360}$ ablation balloon) to deliver radiofrequency energy to the metaplastic esophageal epithelium. Patients are treated with proton pump inhibitors (PPIs) to control acid reflux, and the ablated columnar epithelium heals with the growth of new (neo)squamous epithelium. In $30-46 \%$ of patients, treatment with the $\mathrm{HALO}^{360}$ balloon leaves behind visible foci of Barrett's metaplasia, which can be eradicated with a smaller, endoscope-mounted ablation device (the HALO $^{90}$ ablation catheter) [2]. Using both devices, RFA can safely eliminate all visible evidence of Barrett's metaplasia in up to $98 \%$ of patients [3], and RFA has been shown to prevent the progression from high-grade dysplasia to cancer in Barrett's esophagus in a randomized, controlled trial [4].

In a recent medical position statement, the American Gastroenterological Association has recommended endoscopic eradication therapy for the treatment of patients with confirmed high-grade dysplasia in Barrett's esophagus, but not for the general population of patients with non-

\section{S. J. Spechler $(\bowtie)$}

Division of Gastroenterology, VA North Texas Healthcare

System, The University of Texas Southwestern Medical Center, 4500 South Lancaster Road, Dallas, TX 75216, USA

e-mail: SJSpechler@AOL.com dysplastic Barrett's esophagus [5]. Noting the safety and apparent efficacy of RFA, however, some authorities feel that these guidelines are too restrictive, and argue that virtually all patients with Barrett's esophagus should be treated with RFA, irrespective of dysplasia [6]. El-Serag and Graham recently have argued that, intellectually, ablation for Barrett's esophagus is the same as routine colonoscopic polypectomy for colorectal polyps, and that the practice of limiting endoscopic ablation only to Barrett's esophagus with dysplasia or early cancer is like limiting polypectomy only to polyps that are large or already malignant [7].

Arguments that RFA should be used to treat patients with non-dysplastic Barrett's esophagus are based on the premise that the procedure is effective at decreasing the long-term risk of esophageal cancer. However, it is important to appreciate that no study yet has established that efficacy. Rather, efficacy in preventing cancer in nondysplastic Barrett's esophagus is inferred from short-term studies showing that RFA prevents the progression from high-grade dysplasia to cancer, and from studies showing that RFA can eliminate visible evidence of Barrett's metaplasia for up to 5 years. Unfortunately, elimination of visible evidence of Barrett's metaplasia does not establish that the cancer risk has been eliminated because of two unresolved issues: (1) The frequency with which RFA buries metaplastic glands with malignant potential under a layer of neosquamous epithelium (where they are hidden from the endoscopist) is not clear and, (2) the frequency with which Barrett's metaplasia recurs after initial complete eradication is not clear. In this issue of Digestive Diseases and Sciences, Vaccaro et al. shed some light on the issue of recurrent Barrett's metaplasia [8].

The investigators studied 47 patients who had RFA for Barrett's esophagus, mostly with dysplasia, and who had 
complete eradication with no intestinal metaplasia detected on the first post-ablation endoscopic examination [8]. During all post-ablation endoscopies, four biopsy specimens were taken from columnar epithelium at the gastroesophageal junction (GEJ) just distal to the neosquamocolumnar junction, and biopsies were taken from any areas that appeared suspicious for recurrent Barrett's metaplasia. During a follow-up period ranging from 5 to 38 months, intestinal metaplasia was detected in 15 patients (32\%); at 1 year, the cumulative incidence of intestinal metaplasia was $26 \%$. Four patients $(9 \%)$ had dysplasia found in their recurrent metaplasia, all in biopsy specimens from the GEJ.

The frequency of recurrent Barrett's metaplasia in this study is considerably higher than that observed in three similarly designed studies, which described recurrent metaplasia for only $0-8 \%$ of patients who had complete eradication of Barrett's esophagus by RFA [9-11]. The reason for the disparities among these studies is not clear, but may be related to differences in the surveillance biopsy protocols. Vaccaro's study protocol mandated that biopsy specimens were taken from the GEJ just below the neosquamocolumnar junction, whereas only one of the three aforementioned studies (a small series involving only 12 patients) mandated such biopsies [9].

The esophagus at the GEJ is especially vulnerable to acid-associated injury. The most distal segment of the squamous-lined esophagus is exposed to acid for more than $10 \%$ of the day, even in normal individuals [12]. On the gastric side of the GEJ, the columnar epithelium is exposed for long periods to a pocket of acid that escapes the buffering effects of ingested food, and to potentially genotoxic concentrations of nitric oxide generated from dietary nitrate $[13,14]$. Biopsy specimens taken across the native squamocolumnar junction (the Z-line) in the distal esophagus usually show chronic inflammation involving the squamous epithelium, columnar epithelium, or both [15]. Chronic inflammation is the soil for metaplasia, and biopsy specimens from the Z-line reveal intestinal metaplasia, like that of Barrett's esophagus, in approximately $15 \%$ of unselected patients in general endoscopy units [16]. If metaplasia is to recur anywhere, the GEJ seems the most likely location. It might be argued that, after RFA, patients are treated with PPIs, which should limit acid-peptic injury. PPIs reduce (but do not eliminate) gastric acid production, however, and patients with Barrett's esophagus frequently have abnormal acid reflux even on high-dose PPI therapy [17].

It is not clear whether biopsy specimens taken just below the neo-squamocolumnar junction come from the esophagus or the stomach. The location of the neo-squamocolumnar junction varies depending on the distal extent of the RFA. It is difficult to locate the GEJ with precision, especially in patients with Barrett's esophagus who often have large hiatal hernias [18]. If the RFA does not extend completely into the stomach, then foci of Barrett's metaplasia may be left behind in the most distal esophagus, which might mistakenly be assumed to represent stomach. Alternatively, if the RFA extends below the GEJ into the stomach, then intestinal metaplasia found in biopsy specimens at the neo-squamocolumnar junction may represent gastric intestinal metaplasia (e.g., due to Helicobacter pylori gastritis), which appears to be less predisposed to malignancy than Barrett's metaplasia [18]. It is also not possible to determine whether intestinal metaplasia found at the neo-squamocolumnar junction represents new metaplasia or residual metaplasia that was not eradicated by RFA. The patients in Vaccaro's study had at least one post-ablation endoscopy in which biopsy specimens at the neo-squamocolumnar junction showed no metaplasia, but metaplasia may have been present and simply missed due to biopsy sampling error.

Vaccaro and his colleagues have shown that intestinal metaplasia can be found surprisingly frequently at the GEJ in patients who had what appeared to be complete eradication of Barrett's esophagus by RFA. It is not clear whether this intestinal metaplasia is gastric or esophageal, new or residual, or predisposed to malignancy. The fact that four of 15 patients in this study had dysplastic changes in their newfound intestinal metaplasia suggests that the cancer risk might be substantial. The authors propose that extension of RFA into the stomach, 1-2 cm distal to the GEJ, might help to prevent this problem of recurrent metaplasia. However, if the metaplasia is new rather than residual, then extending RFA below the GEJ might exacerbate the problem by moving the neo-squamocolumnar junction into the stomach where acid exposure would be even greater.

If one accepts the argument that ablation for Barrett's esophagus is intellectually the same as routine polypectomy for colorectal polyps during colonoscopy, then one might argue that recurrence of intestinal metaplasia after RFA is no different than recurrence of colorectal polyps after polypectomy. Colorectal polyps are expected to recur frequently, which is why surveillance colonoscopy is advised. During surveillance colonoscopy, however, recurrent polyps are visible lesions that are removed immediately. After RFA for Barrett's esophagus, Vaccaro has shown that intestinal metaplasia can recur in almost one-third of patients within 3 years and, in most cases, this recurrence is not visible and is detected only as the result of biopsy sampling at the GEJ. Does this mean that patients with recurrent metaplasia in those biopsy samples will require another endoscopy to administer more RFA, and perhaps another endoscopy after that to document that the recurrent metaplasia was eradicated? 
All of the unanswered questions raised by Vaccaro's study must at least temper enthusiasm for the wholesale application of RFA to treat the millions of patients with non-dysplastic Barrett's esophagus in Western countries. Such patients cannot yet be assured that RFA substantially reduces their long-term risk of cancer, and the procedure does not eliminate the need for surveillance endoscopy. A discussion of the profound economic implications of routine ablation of Barrett's esophagus is beyond the scope of this editorial but, without more evidence of benefit, it does not seem wise to adopt this costly practice at this time. We need to start a randomized, controlled trial to establish the risks and benefits of RFA for patients with nondysplastic Barrett's esophagus, the sooner the better.

Acknowledgments This work was supported by the Office of Medical Research, Department of Veterans Affairs, and by the National Institutes of Health R01-CA134571.

\section{References}

1. Spechler SJ, Sharma P, Souza RF, Inadomi JM, Shaheen NJ. American Gastroenterological Association technical review on the management of Barrett's esophagus. Gastroenterology. 2011; 140:e18-52.

2. Spechler SJ, Souza RF. Stem cells in Barrett's esophagus: HALOs or horns? Gastrointest Endosc. 2008;68:41-43.

3. Fleischer DE, Overholt BF, Sharma VK, et al. Endoscopic ablation of Barrett's esophagus: a multicenter study with 2.5-year follow-up. Gastrointest Endosc. 2008;68:867-876.

4. Shaheen NJ, Sharma P, Overholt BF, et al. Radiofrequency ablation in Barrett's esophagus with dysplasia. $N$ Engl J Med. 2009;360:2277-2288.

5. AGA Institute Medical Position Panel. American Gastroenterological Association medical position statement on the management of Barrett's esophagus. Gastroenterology. 2011;140:10841091.
6. Fleischer DE, Odze R, Overholt BF, et al. The case for endoscopic treatment of non-dysplastic and low-grade dysplastic Barrett's esophagus. Dig Dis Sci. 2010;55:1918-1931.

7. El-Serag HB, Graham DY. Routine polypectomy for colorectal polyps and ablation for Barrett's esophagus are intellectually the same. Gastroenterology. 2011;140:386-388.

8. Vaccaro BJ, Gonzalez S, Poneros JM, Stevens PD, Capiak KM, Lightdale CJ, Abrams JA. Detection of intestinal metaplasia after successful eradication of Barrett's esophagus with radiofrequency ablation. Dig Dis Sci. (Epub ahead of print). doi:10.1007/s10620011-1680-4

9. Gondrie JJ, Pouw RE, Sondermeijer CM, et al. Effective treatment of early Barrett's neoplasia with stepwise circumferential and focal ablation using the HALO system. Endoscopy. 2008;40:370-379.

10. Fleischer DE, Overholt BF, Sharma VK, et al. Endoscopic radiofrequency ablation for Barrett's esophagus: 5-year outcomes from a prospective multicenter trial. Endoscopy. 2010;42:781-789.

11. Shaheen NJ, Fleischer DE, Eisen GM, et al. Durability of epithelial reversion after radiofrequency ablation: follow-up of the AIM dysplasia trial. Gastroenterology. 2010;138:A92.

12. Fletcher J, Wirz A, Henry E, McColl KE. Studies of acid exposure immediately above the gastro-oesophageal squamocolumnar junction: evidence of short segment reflux. Gut. 2004;53:168-173.

13. Iijima K, Henry E, Moriya A, Wirz A, Kelman AW, McColl KE. Dietary nitrate generates potentially mutagenic concentrations of nitric oxide at the gastroesophageal junction. Gastroenterology. 2002;122:1248-1257.

14. McColl KE, Clarke A, Seenan J. Acid pocket, hiatus hernia and acid reflux. Gut. 2010;59:430-431.

15. Wang HH, Zeroogian JM, Spechler SJ, Goyal RK, Antonioli DA. Prevalence and significance of pancreatic acinar metaplasia at the gastroesophageal junction. Am J Surg Pathol. 1996;20:1507-1510.

16. Spechler SJ. The columnar lined oesophagus: a riddle wrapped in a mystery inside an enigma. Gut. 1997;41:710-711.

17. Spechler SJ, Sharma P, Traxler B, Levine D, Falk GW. Gastric and esophageal $\mathrm{pH}$ in patients with Barrett's esophagus treated with three esomeprazole dosages: a randomized, double-blind, crossover trial. Am J Gastroenterol. 2006;101:1964-1971.

18. Spechler SJ. The role of gastric carditis in metaplasia and neoplasia at the gastroesophageal junction. Gastroenterology. 1999; 117:218-228. 